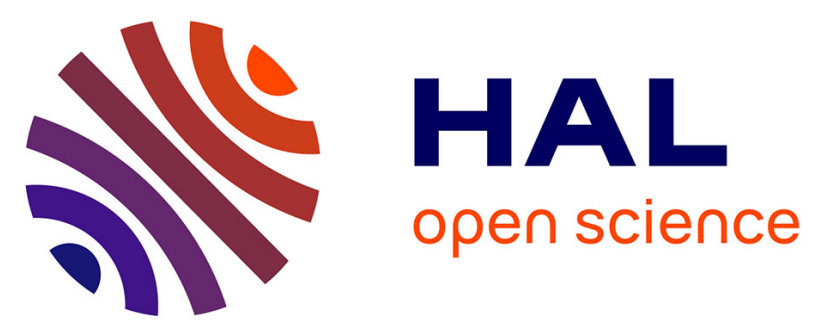

\title{
Assessment of Compressive Raman versus Hyperspectral Raman for Microcalcification Chemical Imaging
}

Camille Scotté, Hilton B de Aguiar, Didier Marguet, Ellen Marie Green, Pascaline Bouzy, Sébastien Vergnole, Charles Peter Winlove, Nicholas Stone, Herve Rigneault

\section{To cite this version:}

Camille Scotté, Hilton B de Aguiar, Didier Marguet, Ellen Marie Green, Pascaline Bouzy, et al.. Assessment of Compressive Raman versus Hyperspectral Raman for Microcalcification Chemical Imaging. Analytical Chemistry, 2018, 90 (12), pp.7197-7203. 10.1021/acs.analchem.7b05303 . hal-01816013

\section{HAL Id: hal-01816013 \\ https://hal-amu.archives-ouvertes.fr/hal-01816013}

Submitted on 4 Jul 2018

HAL is a multi-disciplinary open access archive for the deposit and dissemination of scientific research documents, whether they are published or not. The documents may come from teaching and research institutions in France or abroad, or from public or private research centers.
L'archive ouverte pluridisciplinaire HAL, est destinée au dépôt et à la diffusion de documents scientifiques de niveau recherche, publiés ou non, émanant des établissements d'enseignement et de recherche français ou étrangers, des laboratoires publics ou privés. 


\title{
Assessment of compressive Raman versus hyperspectral Raman for microcalcification chemical imaging
}

\author{
Camille Scotté ${ }^{1}$, Hilton B. de Aguiar ${ }^{2, *}$, Didier Marguet ${ }^{3}$, Ellen Marie Green ${ }^{4}$, Pascaline Bouzy ${ }^{4}$, \\ Sébastien Vergnole ${ }^{5}$, Charles Peter Winlove ${ }^{4}$, Nicholas Stone ${ }^{4}$ and Hervé Rigneault ${ }^{1, *}$ \\ ${ }^{1}$ Aix Marseille Univ, CNRS, Centrale Marseille, Institut Fresnel, Marseille, France \\ ${ }^{2}$ Département de Physique, Ecole Normale Supérieure / PSL Research University, CNRS, 24 rue Lhomond, 75005 Paris, \\ France \\ ${ }^{3}$ Aix-Marseille Université, INSERM, CNRS, Centre d'Immunologie de Marseille-Luminy, Marseille, France \\ ${ }^{4}$ School of Physics and Astronomy, University of Exeter, Exeter, UK \\ ${ }^{5}$ HORIBA Scientific, Villeneuve d'Ascq, France \\ *Corresponding authors: h.aguiar@phys.ens.fr, herve.rigneaul@fresnel.fr
}

\begin{abstract}
We experimentally implement a compressive Raman technology (CRT) that incorporates chemometric analysis directly into the spectrometer hardware by means of a digital micromirror device (DMD). The DMD is a programmable optical filter on which optimized binary filters are displayed. The latter are generated with an algorithm based on the Cramer-Rao lower bound. We compared the developed CRT microspectrometer with two conventional state-of-the-art Raman hyperspectral imaging systems on samples mimicking microcalcifications relevant for breast cancer diagnosis. The CRT limit of detection significantly improves, when compared to the CCD based system, and CRT ultimately allows 100x and 10x faster acquisition speeds than the CCD and EMCCD-based systems, respectively.
\end{abstract}

Swift chemical analysis with hyperspectral imaging would be a milestone for techniques such as reflectance or vibrational spectroscopy. The latter include the widely used spontaneous Raman spectroscopy which, through inelastic scattering of light due to molecular vibrational modes, enables chemical systems characterization with high molecular selectivity. Nevertheless, the effectiveness of this simple process is limited by the extremely weak Raman cross-section which implies long acquisition times and limits its further implementation for invivo imaging or dynamic processes.

Conventional implementation of hyperspectral imaging acquisition requires the spectral dispersion of the Raman scattered light onto an array detector, typically a charged couple device (CCD) or an electron multiplying CCD (EMCCD), for each spatial position of the sample. In many situations, the aim is actually to quantify or classify the chemical species given their spectra, and numerous advanced multivariate statistical techniques have been developed to efficiently solve this spectral unmixing problem. (1-2)

Nevertheless, measuring a complete vibrational Raman spectrum per spatial pixel, coupled to the weak Raman signal and detector array noise, leads to lengthy acquisitions and to the generation of overwhelmingly large data sets. For instance, mapping an area of $100 \times 100$ pixels (each spectrum consisting of 1000 spectral pixels) with a state-of-the art spectroscopic 16-bits EMCCD takes at least $10 \mathrm{~s}$ and requires $19 \mathrm{Mb}$ of storage. One strategy to make the technique faster is to perform wide-field imaging instead of point scanning (see, for instance, refs (3-5)). However, those methods show limitations in terms of resolution and reconstruction time. Another approach relies on the fact that acquiring complete vibrational spectra may be inefficient when the information of interest (species proportions or class) is confined in a low dimensional basis.

The latter considerations are at the origin of alternative approaches based on compressive Raman technology (CRT) that have recently been proposed (6-12) for quantification or classification of know species. The idea is to incorporate chemometric analysis directly into the spectrometer hardware. Thus, the measurement is designed to directly probe quantities of interest (species proportions or class) instead of deducing them from complete hyperspectral measurements. Typically, a programmable optical filter (e.g. DMD, digital micromirror device) displays optimized patterns to select wavelength combinations that efficiently estimate the quantities of interest. Corresponding photons are combined in a single-channel detector that replaces the detector array used in conventional hyperspectral imaging, with significant improvement in acquisition speed. Based on the theoretical work of Buzzard and Lucier (10), Wilcox et al. implemented experimentally $(8,9,11)$ a CRT strategy through optimal binary filters that were designed by minimizing the estimation error of the variables of interest (10). The method was validated on microspectroscopy in a 
mixture of glucose and fructose, demonstrating discrimination with as low as $\sim 10$ Raman photons per pixel, corresponding to pixel dwell time of $\sim 30 \mu \mathrm{s}$.

One of the main motivations for CRT is to boost acquisition speed in order to ultimately enable in-vivo imaging or characterization of dynamic processes. However, no CRT applied to samples of biological interest has been reported to date (8-10), nor has its performance been assessed compared to conventional hyperspectral imaging. The scope of the present paper is to fill this gap. In this context, we chose a biological system particularly relevant for breast cancer diagnosis - breast microcalcifications. Indeed, microcalcifications in the breast are an important indicator for cancer and are often considered in diagnosis. Several studies suggested that microcalcifications reflect the physiological state of surrounding tissue and that their chemical composition is related to cancer development (13-17). It has been shown that Raman spectroscopy is particularly useful for determining microcalcifications chemical composition correlated with the cancer state (17).

In this paper we compare CRT to state-of-the-art conventional hyperspectral imaging with CCD and EMCCD cameras and assess its capability on microcalcification discrimination that are relevant to detect breast cancer in-vivo (13-17). Another novelty of our approach is the CRT filter design that combines simplicity and precision close to standard approaches using complete spectral acquisition Raman spectroscopy (18).

\section{EXPERIMENTAL SECTION}

\section{COMPRESSIVE RAMAN TECHNOLOGY (CRT)}

The context of this study is chemical quantitation where we wish to estimate the proportions of mixed chemical species whose pure Raman spectra are known. To do so with conventional spectroscopy, a full spectrum of the mixture has to be measured. Instead, the basic concept of CRT consists in performing the spectral detection through few filters to directly probe the species proportions. This approach requires an appropriate spectrometer design as well as adequate analytical developments.

\section{Experimental setup}

The compressive Raman spectrometer is similar to a conventional spectrometer in which the detector array (CCD or EMCCD) is replaced by a single channel detector combined with a digital micromirror device (DMD). On the latter, spectral filters are displayed, leading to accurate and precise estimation of chemical proportions (section Filter Design and Estimation). The main parts of the CRT spectrometer used in this study are shown in the simplified layout in Figure 1. More details are given in Supporting Information (SI, Fig S-1).

The sample was excited with a $532 \mathrm{~nm} \mathrm{CW}$ laser. The backscattered light is collected by the spectrometer entrance slit, dispersed with a grating and focused onto a DMD (19). The micromirrors were horizontally binned (4 mirrors/pixel), and vertically fully binned. The columns of pixels were then pivoted either towards the detector (ON position) or away from the detector (OFF position), and the selected wavelengths are combined onto a single channel photo-multiplier tube (PMT) working in photon counting regime. One filter displayed on the DMD is associated with one combination of wavelengths and thus with one measurement. A full Raman spectrum (FRS) can also be acquired by raster scanning the DMD array with only one column of mirrors $\mathrm{ON}$ at a time. The spectral resolution (FWHM) was $40 \mathrm{~cm}^{-1}$ and the spatial resolution was 1.4 $\mu \mathrm{m}$.

For the validation experiment on beads (section Technique Validation), laser power at the sample plane was $4 \mathrm{~mW}$, the region of interest (ROI) $60 \times 60 \mu \mathrm{m}^{2}$ and the spatial pixel size $0.65 \mu \mathrm{m}$. For the experiments on microcalcifications powders (section CRT Results on Microcalcifications Powders Samples), the laser power at the sample plane was $60 \mathrm{~mW}$, the ROI was $80 \times 80 \mu \mathrm{m}^{2}$ and spatial pixel size $0.75 \mu \mathrm{m}$. Images were acquired using a high resolution piezo stage. The pixel dwell time of all raw images was $4 \mathrm{~ms}$. We assess the CRT performance (section Comparison of CRT and Hyperspectral Limits of Detection: Results and Discussion) by decreasing the number of detected photons (by reducing the excitation laser power on the sample).

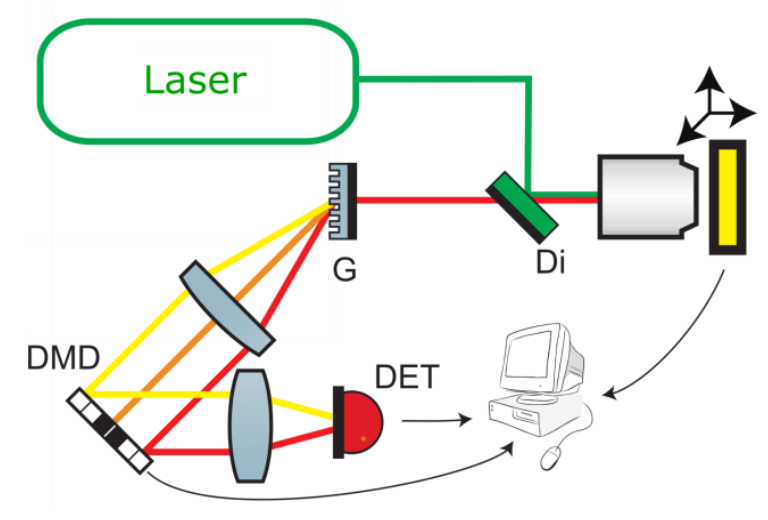

Figure 1. Simplified experimental layout for compressed Raman microspectroscopy Di: dichroic mirror, G: grating, DMD: digital micromirror device, DET: single channel PMT detector working in photon counting regime.

\section{Filter design and estimation}

At the core of the CRT lies the design of the filters to be displayed on the DMD to allow efficient estimation of species proportions.

The filters design is based on maximizing the precision of the species proportions estimate $\hat{\mathbf{z}}=\left(\widehat{\mathbf{z}_{1}}, \ldots, \widehat{\mathbf{z}_{Q}}\right)^{T}$, with $Q$ the number of species and ${ }^{\mathbf{T}}$ the transpose operation. Considering an unbiased estimator and random variables (photon counts) following a Poisson distribution (Figure S-2), the design of filters is achieved by minimizing the trace of the Cramer-Rao lower bound (CRB) matrix (18). The CRB is the lower bound on the variance of any unbiased estimator. The algorithm inputs are: the pure chemical species Raman spectra, equal initial species proportions with equal exposure times for each filter (SI, section A).

If $M$ filters $F$ are projected onto the DMD, the $M$ photon measurements $\mathbf{n}$ with mean $\boldsymbol{\mu}=\langle\boldsymbol{n}\rangle$ are given in (18) by:

$$
\mu=\boldsymbol{G} \mathbf{z}
$$


The $M \times Q$ matrix $\boldsymbol{G}$ represents the projection of the filters $F$ on the pure known spectra and is thus related to the wavelengths signals filtering (SI, eq S.4). The proportions are then simply retrieved through linear estimation:

$$
\hat{z}=\left[G^{T} G\right]^{-1} G^{T} n
$$

We show in (18) that the linear estimation method is efficient, i.e. unbiased with variance equals to the $\mathrm{CRB}$.

The method for filters calculation is similar to the one developed in (10). Indeed, our method minimizes the trace of the CRB while the method in (10) minimizes the trace of the covariance matrix $\Gamma$. The two methods are equivalent for $M=Q$ as shown in (18). The difference relies on the numerical strategies for calculating the filters. We generate a random binary matrix and replace its values while trace $(\boldsymbol{C R B})$ is decreased (see more details in SI, section A and (18)). The algorithm of (10) could have been used in this paper with expected similar results. Differences between the two algorithms are expected to be negligible in the scope of this paper where CRT is compared to a different method (hyperspectral imaging).

Finally, it is worth noting that filter optimization and proportion estimation can be performed with simple and very computationally tractable methods.

\section{Technique validation}

The CRT for microspectroscopy proportion estimation was first validated on bead samples. We used two types of latex beads spatially distributed on a $\mathrm{CaF}_{2}$ slide: melamine resin (MR) with $12 \mu \mathrm{m}$ diameter (Sigma) and polystyrene (PS) with $30 \mu \mathrm{m}$ diameter (Sigma). Their resonances show some overlap (dot product of normalized spectra is 0.72 ) as can be seen on Figure 2.

The reference spectra (Fig 2.Left) were acquired by raster scanning the DMD. In all CRT experiments, spectra are nonnormalized and non-background subtracted. The background was not taken into account in order to have a simple model with a minimum number of filters. Each reference spectrum is an average of 3 spectra taken at different spatial points on the respective sample (MR or PS), each for an integration time of $1 \mathrm{~s}$ per spectral pixel. The reference spectra serve as an input to generate the filters (F1 and F2) displayed on Figure 2.

The sample entire ROI was scanned sequentially with filters F1 and F2 displayed on the DMD. The raw data forms two images: in each pixel of each image is the number of photons measured by projecting one filter onto the sample (Figure $S$ 9). To obtain the average total number of detected photons per spatial pixel, we simply sum the numbers of photons detected with the two filters and average over the species pixels (SI, section F). This way, we obtain an average of 300 photons on MR pixels and 800 photons on PS pixels.

Post-processing consisted in estimating the proportion $\hat{\boldsymbol{z}}$ of each species in each pixel, as given by eq (2). Note that since this sample consists of pure species, the true proportion coefficients $Z_{i}$ should take the values $\{0,1\}$. The estimated proportions maps are shown in Figure 2, right panel. The maps were constructed with setting the raw estimated proportions coefficients to lie in the interval [0 1]. We clearly see on the top map that on the PS pixels, the estimated proportions are close to
$100 \%$, whereas MR pixels have proportions close to $0 \%$. The opposite case is observed on the bottom map, on which only the MR pixels show proportions close to $100 \%$. The discrimination image was obtained through proportion map normalization as given in equations (S.7) and (S.8).

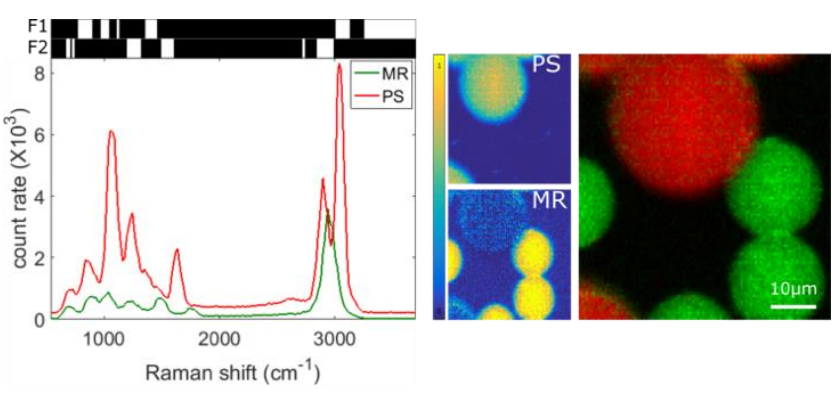

Figure 2. Left: Reference spectra of polystyrene (PS) and melamine resin (MR) and their associated optimized spectral filters F1 and F2 (top). Right: proportion coefficients maps (estimation of $\widehat{\boldsymbol{z}}$ ) of PS (top) and MR (bottom), and the associated RGB image (MR: green, PS: red). Total integration time per pixel (F1 plus $\mathrm{F} 2)$ is $8 \mathrm{~ms}$.

\section{CONVENTIONAL HYPERSPECTRAL IMAGING}

We want to assess the relevance of the CRT compared to conventional hyperspectral imaging. We chose to adopt an engineer-oriented approach: to perform hyperspectral imaging on two available state-of-the-art spectrometers, each equipped with different detectors (CCD and EMCCD) and to compare with our custom-built CRT.

\section{Intrumentation}

The first Raman imaging system was the WITec Alpha300R equipped with a CCD (DV401A-BV, Andor). The second system was the HORIBA LabRAM HR Evolution equipped with an EMCCD (Synapse EM, HORIBA). In both cases, the excitation source was a $532 \mathrm{~nm} \mathrm{CW}$ laser; the sample was placed in the focal plane and scanned over a chosen ROI. One spectrum per pixel was acquired, resulting in a hyperspectral image.

The experiments on the WITec were conducted in the low readout noise regime (slow $\mathrm{A} / \mathrm{D}$ rate). In this mode, the minimum achievable integration time per spectra is $43 \mathrm{~ms}$, regardless of the signal level. One image of $100 \times 100$ pixels could then be acquired in about $7 \mathrm{~min}$ at best.

On the HORIBA system, high EMCCD gain was chosen so that the readout noise is negligible even at the fastest available $\mathrm{A} / \mathrm{D}$ rate $(3 \mathrm{MHz})$. The system was used in the fastest scanning mode, so that one spectrum could be acquired in about 2 ms. Thus, one image of 100x100 pixels could be acquired in about $20 \mathrm{~s}$ at best in the chosen mode.

Spatial and spectral resolutions are not the same in the systems, and were chosen according to available settings, gratings and objectives. Further details about experimental conditions, modes, and systems specifications are given in SI, section B. The main specifications of the Raman imaging systems and 
their respective sensors, as well as our custom build CRT system are listed in Table S-1.

\section{Proportion estimation}

The first post-processing step consisted in subtracting the baseline level from all spectra. The CCD and EMCCD baseline levels (as well as electronic noise) were assessed from dark frame measurements (SI, section C).

From the resulting hyperspectral images, we want to generate proportion maps (relative amounts of chemical species present in the sample) in order to obtain data comparable with the CRT. In hyperspectral imaging, the full Raman spectrum $\boldsymbol{y}$ is measured in each pixel and we need to estimate the relative amount of chemical species $\boldsymbol{Z}$ present in each spectrum. The pure reference spectra $(\boldsymbol{S})$ are measured independently and averaged on different sample points. Each pixel can be composed of a mixture of pure species in relative proportions:

$$
y=z S
$$

To find the proportions, we use a simple least square estimator (which is unbiased) with solution:

$$
\widehat{\boldsymbol{z}_{L S}}=\left(\boldsymbol{S}^{T} \boldsymbol{S}\right)^{-1} \boldsymbol{S}^{T} \boldsymbol{y}
$$

Estimating proportions leads to one proportion map per species. Since taking into account the background spectrum (from $\mathrm{CaF}_{2}$ slide) was not adding extra acquisition time (as opposed to CRT where one additional filter is generated), the background spectrum was taken into account but not considered as a species in the final images. The steps described in SI, section E were applied to obtain the final images.

\section{ASSESSMENT OF TECHNIQUES ON BIOMEDICAL SAMPLES}

In this section we apply CRT on samples mimicking microcalcifications commonly found in human breast. We then assess the relevance of CRT compared to conventional hyperspectral imaging on this pertinent biological framework.

\section{Background on microcalcifications}

In the breast, two types of microcalcifications are frequently found. Type I microcalcifications, mostly associated with benign tissue, are composed of calcium oxalate $\mathrm{CaC}_{2} \mathrm{O}_{4}$ (e.g. in the monohydrate form - COM). Type II microcalcifications consist of hydroxyapatite (HAP), a mineral form of calcium apatite with lattice unit cell $\mathrm{Ca}_{10}\left(\mathrm{PO}_{4}\right)_{6}(\mathrm{OH})_{2}$. A substitutional defect that is likely to occur in HAP is the substitution of a phosphate group by a carbonated group forming carbonated hydroxyapatite (CHAP) (13-17). Studies (13-17) have shown that carbonate content in CHAP is greater in benign than in malignant breast tissue. Carbonate substitution in CHAP has a Raman spectroscopic signature, manifested by a shift of the resonance at $1048 \mathrm{~cm}^{-1}$ to $1071 \mathrm{~cm}^{-1}$ (carbonate signature) (16). Therefore, distinguishing type I from type II microcalcifications and assessing the carbonate content of type II with spectroscopy is appealing for breast cancer diagnosis.

In this work, we used three synthetic powders mimicking the chemical composition of the microcalcifications COM (Alfa Aesar), HAP (Sigma Aldrich) and HAP with 8.12\% carbonate substitution (Sigma Aldrich) - CHAP (Such highly concentrated CHAP is not believed to be found in the breast but exhibits the same spectral peaks as less concentrated CHAP, with more pronounced carbonate peak at $1071 \mathrm{~cm}^{-1}$ ).

Experiments focused on discriminating HAP and CHAP or HAP and COM samples. They were conducted on the CRT setup and on the two commercial spectrometers.

\section{CRT results on microcalcifications powders samples}

The experiments are initially conducted with the powder samples dispersed onto $\mathrm{CaF}_{2}$ coverslips. We followed-the same method as in the validation example on beads.

Unlike in the latter, the reference spectra of CHAP and HAP are quite similar (dot product of normalized spectra $=0.85$ ) and discriminating those two species is fairly complicated. Their reference spectra were acquired with $0.5 \mathrm{~s}$ integration time and averaged on 4 different spatial points. For the CRT model, we included the background from $\mathrm{CaF}_{2}$ (blue) and a simulated constant background (black) (Figure 3.a). The latter simulates an experimental background and acts as an additive constant for better robustness. More filters are generated and allow the species proportions to be accurately estimated. With the procedure described in SI, section E, a RGB image showing HAP (red) and CHAP (green) is obtained (Figure 3.b).

In the second experiment, we replace CHAP by COM and proceed in the same way. However, the problem is simpler (dot product of normalized spectra $=0.79$ ): the reference spectra were acquired with $0.1 \mathrm{~s}$ integration time on 4 different spatial points, and no background was included into the CRT model (Figure 3.c). This simple two filter projection allows accurate CRT proportion estimation (Figure 3.d).

In a third experiment, we place a $1 \mathrm{~mm}$ piece of chicken breast on a glass coverslip. We then sprinkle sample powders on the chicken tissue. We then acquire the tissue spectrum ( 0.1 integration time on 4 points). However, we don't know where the species of interest in this highly scattering tissue are. Therefore, we re-use the COM and HAP spectra already learnt in the previous experiment without chicken breast (Figure 3.e). After filters generation and projection, we could estimate the species and tissue proportions and obtain an RGB image (Figure 3.f). Though this experiment is simplistic and does not reflect a real sample, it gives an idea of the robustness of the model in presence of tissue background. See SI, section $\mathrm{H}$ for more details on this tissue experiment. 


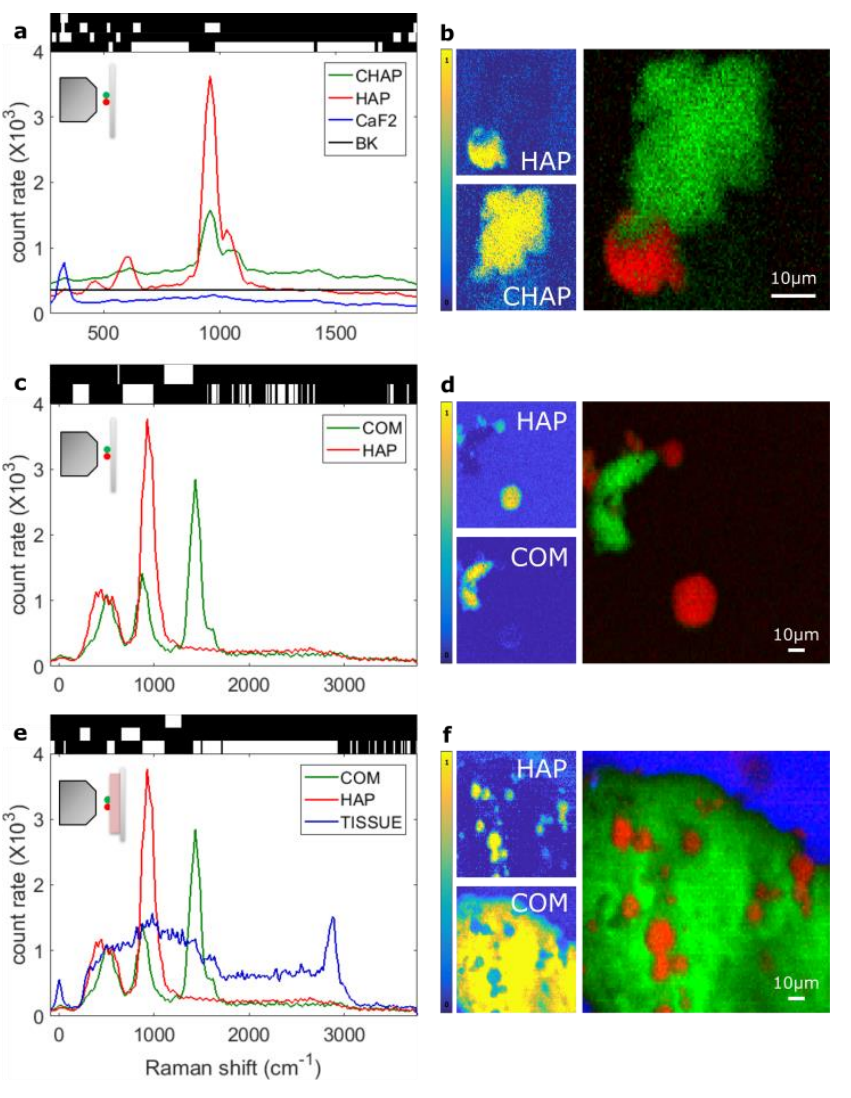

Figure 3. (a): CHAP-HAP on $\mathrm{CaF}_{2}$ coverslip and their reference spectra. $\mathrm{CaF}_{2}$ and a simulated background spectra are included in the model. (c): COM-HAP on a $\mathrm{CaF}_{2}$ coverslip and their reference spectra. (e): COM-HAP on a slice of chicken breast tissue deposited on a glass coverslip. COM and HAP reference spectra learnt in step (c), and tissue reference spectra.

(b), (d) and (f): proportion coefficient maps obtained after estimation and related RGB images. Only the microcalcifications powders proportion maps are shown.

\section{Comparison of CRT and hyperspectral limits of detection: results and discussion}

In this section we compare hyperspectral imaging and CRT techniques for the detection of low concentrations of molecular species such as found in living systems.

In this section we want to gain insight of the systems limit of detection (LOD). By LOD, we mean the minimal Raman signal (integrated over all wavelengths) that must arrive on the detector to be able to achieve species proportion estimation. This photon budget is distributed over the cameras pixels. In opposition, the CRT filters combine the relevant signal spectral components together into a single pixel. Furthermore, only CRT is shot-noise limited at low signal levels (SI, sections C and D). Thus, for equal detectors QE, we expect a better performance of CRT (see explanations SI, section D).

To get the LOD order of magnitudes, experiments were conducted close to the system detection limits with the hyperspectral imaging systems (WITec with CCD and HORIBA with EMCCD) and our custom built CRT. HAP-COM and HAPCHAP powders dispersed on $\mathrm{CaF}_{2}$ substrates were imaged decreasing gradually the laser power on the sample. The clos- est experiment to the LOD is the last experiment until the SNR is too low to estimate proportions successfully. Thus we have access to the interval where the LOD lies for those experiments. We give in the following an upper bound on the LOD.

The proportion coefficient maps were obtained using the equations (4) for hyperspectral imaging and (2) for CRT, with the same spectral range for all systems. The RGB images were then generated. Because the systems have different throughputs, we compared $N_{t o t}$ - average photon number detected per spatial pixel - close the three systems LOD (SI, section D). For CRT, we obtain $N_{\text {tot }}$ by summing the numbers of photons detected after each filter projection. We then average over the species pixels (SI, section F). The number of photoelectrons is the number of photons multiplied by the detector quantum efficiency (QE). For hyperspectral imaging, we don't integrate the full spectrum on each pixel. For a more fair comparison, we generate binary filters with the reference spectra $\boldsymbol{S}$ acquired with the specified systems (SI, section D). We then project those filters (except the filter corresponding to the background) on the hyperspectral data cube and proceed as for CRT. We finally relate $N_{\text {tot }}$ to the numbers of camera counts with equations (S.13) and (S.14).

Figures 4 and 5 present proportion coefficient maps close to the LOD (upper bound) for the COM-HAP and HAP-CHAP (see also SI, section E). We found that for the CCD system, the minimal number of photons lies between 150 and 450 for COM-HAP successful quantitation and 1000 for HAP-CHAP (SI, section D). This is one to two orders of magnitudes higher than for the CRT ( 20 for both COM-HAP and HAP-CHAP). The EMCCD system can do better and the minimal number of photons for successful quantitation is similar to CRT for COM-HAP and one order of magnitude higher for HAPCHAP (Note the evaluation of the photon number for EMCCD is subjected to imprecision coming from the probabilistic nature of the multiplication stage). We also relate the estimated LOD upper bounds to a SNR value on a "decisive" pixel for successful quantitation in SI, section D.

We thus conclude that CRT limit of detection is one to two orders of magnitude lower than CCD equipped system, but similar to EMCCD in view of the imprecision concerning the spectrometers throughput, focusing and sample variability. However, our PMT QE is twice as worse as the camera's, so CRT would show greater advantage if equipped with a single pixel detector with higher quantum efficiency (e.g. avalanche photodiode).

However, regardless of the sensitivity, the CRT pixel dwell time capability is faster than CCD and EMCCD, as described in the next section.
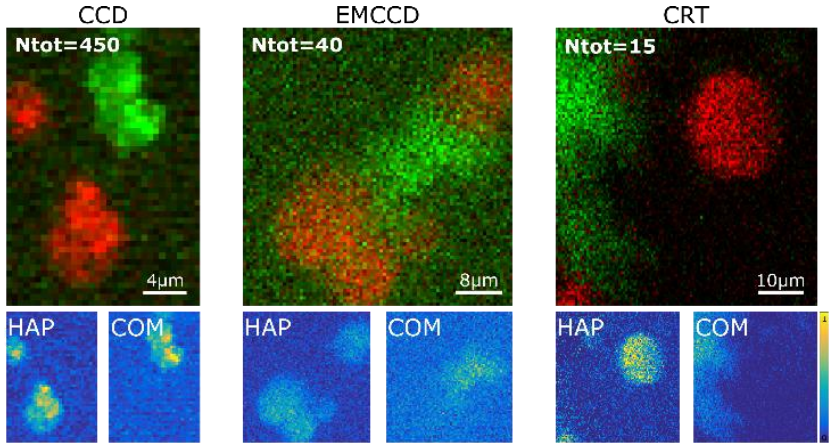
Figure 4. HAP (red) and COM (green) RGB images from the bottom proportion coefficient maps obtained close to the limit of detection of the WITec equipped with CCD (Left), HORIBA LabRAM HR Evolution equipped with EMCCD (Middle), and the custom built CRT equipped with PMT (Right). Ntot is the total average number of photons on species pixels given by equations (S.11) and (S.14).
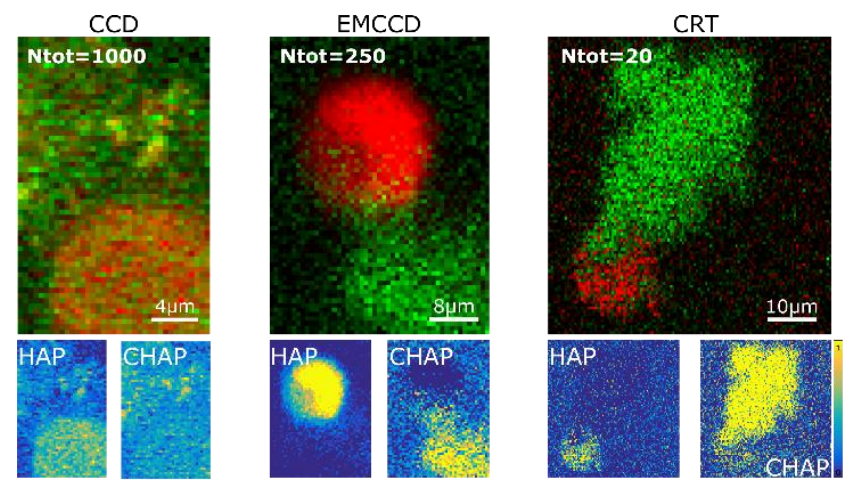

Figure 5. HAP (red) and CHAP (green) RGB images from the coefficient proportion maps obtained close to the limit of detection of the WITec equipped with CCD (Left), HORIBA LabRAM HR Evolution equipped with EMCCD (Middle), and the custom built CRT equipped with PMT (Right). Ntot is the total average number of photons on species pixels given by equations (S.11) and (S.14).

Table 1. Comparison of the limits of detection and fundamental fastest speeds of the instruments

\begin{tabular}{|c|c|c|c|c|}
\hline & & $\begin{array}{l}\text { WITec } \\
+ \text { CCD }\end{array}$ & $\begin{array}{l}\text { HORIBA } \\
+ \text { EMCCD }\end{array}$ & $\begin{array}{l}\text { CRT } \\
+ \text { PMT }\end{array}$ \\
\hline \multicolumn{5}{|c|}{ Low signal regime } \\
\hline \multicolumn{2}{|l|}{ SNR limitation } & $\begin{array}{l}\text { Readout } \\
\text { noise }\end{array}$ & $\begin{array}{l}\text { Excess } \\
\text { noise } \\
\text { Factor } \quad(20- \\
22)\end{array}$ & $\begin{array}{l}\text { Photon } \\
\text { noise }\end{array}$ \\
\hline $\begin{array}{l}\text { Power on sam- } \\
\text { ple }\end{array}$ & $\begin{array}{l}\mathrm{A} \\
\mathrm{B}\end{array}$ & $\begin{array}{l}0.3 \mathrm{~mW} \\
7 \mathrm{~mW}\end{array}$ & $\begin{array}{l}0.4 \mathrm{~mW} \\
4 \mathrm{~mW}\end{array}$ & $\begin{array}{l}2 \mathrm{~mW} \\
4 \mathrm{~mW}\end{array}$ \\
\hline $\begin{array}{l}\text { LOD upper } \\
\text { bound } \\
\text { ( } N_{\text {tot }} \text { photons) }\end{array}$ & $\begin{array}{l}\text { A } \\
\text { B }\end{array}$ & $\begin{array}{l}450 \\
1000\end{array}$ & $\begin{array}{l}40 \\
250\end{array}$ & $\begin{array}{l}15 \\
20\end{array}$ \\
\hline $\begin{array}{l}\text { LOD upper } \\
\text { bound } \\
\text { ( } N_{\text {tot }} Q E \text { pho- } \\
\text { toelectrons) }\end{array}$ & $\begin{array}{l}\text { A } \\
\text { B }\end{array}$ & $\begin{array}{l}427 \\
950\end{array}$ & $\begin{array}{l}36 \\
225\end{array}$ & $\begin{array}{l}6 \\
8\end{array}$ \\
\hline \multicolumn{5}{|c|}{ High signal regime } \\
\hline SNR limitation & & $\begin{array}{l}\text { Photon } \\
\text { noise }\end{array}$ & $\begin{array}{l}\text { Excess } \\
\text { noise factor }\end{array}$ & $\begin{array}{l}\text { Photon } \\
\text { noise }\end{array}$ \\
\hline$t_{\min }$ & & $10 \mathrm{~ms}$ & $1 \mathrm{~ms}$ & $<100 \mu \mathrm{s}$ \\
\hline$t_{\text {mintotal }}$ & & $100 \mathrm{~s}$ & $10 \mathrm{~s}$ & $<1 \mathrm{~s}$ \\
\hline
\end{tabular}

A: HAP-COM experiment; B: HAP-CHAP experiment. LOD: minimum photon budget for proportion estimation. The number of photoelectrons is related to the number of detected photons by the quantum efficiency QE. $\boldsymbol{t}_{\text {min }}$ : Minimum achievable pixel dwell time (not scanning speed limited), $\boldsymbol{t}_{\text {min total }}$ total: Minimum achievable total scan time for 100x100 pixels image.

Comparison of the fundamental speed limits of the techniques

We compare here the fastest speeds achievable by the three systems when the measurements are not limited by the SNR. As shown in Table 1, the WITec + CCD system can at best achieve speeds of the order of $10 \mathrm{~ms} / \mathrm{spectrum}$, while the HORIBA + EMCCD system can reach speeds of the order of 1 $\mathrm{ms} / \mathrm{spectrum}$. The speed limit is due to the readout speed of those cameras. Since hyperspectral imaging is performed through point scanning (one spectrum per pixel), the acquisition of a FOV of 100x100 pixels required at minima respectively $100 \mathrm{~s}$ and $10 \mathrm{~s}$. Afterwards, multivariate techniques like PCA, MCR or least-square estimation can be performed in post-processing to obtain images allowing discriminating species.

With CRT, once the reference spectra are learnt and the filters generated, photon counting is performed in each pixel (instead of the standard spectral acquisition in each pixel). Therefore, the scanning speed is only fundamentally limited by the maximum count rate of the detector (one photon each 100 ns). In practice in a scanning microscope, it is limited by the scanning system speed ( $<1 \mu$ s per pixel for galvanometric mirrors).

From the recorded data (Figure 4, 5), we conclude that CRT can perform Raman imaging with successful COM/HAP and HAP/CHAP detection in less than $100 \mu \mathrm{s}$ pixel dwell time, compared with the minimum of $10 \mathrm{~ms}$ and $1 \mathrm{~ms}$ pixel dwell time for the WITec + CCD and HORIBA + EMCCD system, respectively, bringing a x100 to x10 speed improvement for CRT Raman imaging

\section{CONCLUSION}

We have evaluated compressive Raman technology (CRT) using a new Cramer-Rao lower bound (CRB) based algorithm and compared it with state-of-the-art commercially available hyperspectral Raman systems. Both CCD and EMCCD based hyperspectral Raman systems have been considered. We have performed our evaluation on microcalcifications powders that show biological relevance in the context of human breast cancer.

In the high signal regime where all systems can perform successful species quantitation, CRT with fast scanning scheme is x100 times faster the CCD based system and x10 times faster than the EMCCD based system. Whereas a 100x100 pixels image requires about $100 \mathrm{~s}$ with CCD and $10 \mathrm{~s}$ with EMCCD, CRT would take less than a second.

In the low signal regime when noise limits the detection, we found that CRT has a limit of detection similar to EMCCD and up to 100x higher than CCD. However, for an equal limit of detection, CRT pixel dwell time can be 10 times faster than EMCCD and 100 times faster than CCD. 
It is worth noting that our CRT custom build spectrometer is far from being optimal in terms of brightness and detector QE and we anticipate that the superiority of CRT can be further improved.

An interesting follow-up to this work would be to perform CRT experiments on real microcalcifications embedded into human breast tissue.

Overall, our results open interesting perspectives in the field of high speed Raman imaging and fast Raman spectroscopy on dynamical systems - as the DMD rate $(22 \mathrm{kHz})$ enables CRT estimation at $\mathrm{kHz}$ frequency.

\section{ASSOCIATED CONTENT}

\section{Supporting Information}

See Supporting Information (SI) for further content: CRT theory, instrumentation, detectors characteristics, signal to noise ratio and limit of detection considerations, post-processing details, experimental details on the CRT experiment in the presence of background.

\section{AUTHOR INFORMATION}

\section{Corresponding Authors}

* h.aguiar@phys.ens.fr, herve.rigneaul@fresnel.fr

\section{Author Contributions}

The manuscript was written through contributions of all authors. / All authors have given approval to the final version of the manuscript.

\section{ACKNOWLEDGMENT}

The authors acknowledge Frédéric Galland and Philippe Réfrégier (Institut Fresnel) for conducting the theoretical work published in (18) and for sharing ideas through inspiring discussions. The authors also acknowledge stimulating discussion and support from Olivier Piot (Univ Reims Champagne Ardennes).

C. S. has received funding from the European Union's Horizon 2020 research and innovation program under the Marie Skłodowska-Curie grant agreement No713750. The project received financial support from: the Regional Council of Provence- Alpes-Côte d'Azur, A*MIDEX ( ${ }^{\circ}$ ANR- 11-IDEX-0001-02), France Bio Imaging (ANR-10-INSB-04-01), France Life Imaging project (ANR-11-INSB-0006), INSERM: PC201508. H. B. A. is supported by LabEX ENS-ICFP: ANR-10-LABX-0010/ANR-10-IDEX0001-02 PSL.

\section{REFERENCES}

1. Felten, J.; Hall, H.; Jaumot, J.; Tauler, R.; De Juan, A; Gorzsás, A. Nat. Protoc. 2015, 10(2), p.217.

2. M. Wang, L. and Zhao, C. Hyperspectral Image Processing. Springer, 2016.

3. Thompson, J. V.; Bixler, J. N.; Hokr, B. H.; Noojin, G. D.; Scully, M. O.; Yakovlev, V. V. Opt. Lett. 2017, 42.11, 21692172.

4. Galvis-Carreño, D.F.; Mejía-Melgarejo, Y.H.; ArguelloFuentes, H. Dyna. 2014, 81.188, 116-124.

5. Wadduwage, D. N.; Singh, V. R.; Choi, H.; Yaqoob, Z.; Heemskerk, H.; Matsudaira, P.; So, P. T. Optica. 2017, 4.5, 546-556.

6. Pavillon, N.;N. I. Smith. Opt. Express. 2016, 24.26, 3003830052.

7. Davis, B. M.; Hemphill, A. J.; Cebeci Maltaş, D.; Zipper, M. A.; Wang, P.; Ben-Amotz, D. Anal. Chem. 2011, 83.13, 5086-5092.

8. Wilcox, D. S.; Buzzard, G. T.; Lucier, B. J.; Wang, P.; BenAmotz, D. Anal. Chim. Acta. 2012, 755, 17-27.

9. Wilcox, D. S.; Buzzard, G. T.; Lucier, B. J.; Rehrauer, O. G.; Wang, P.; Ben-Amotz, D. Analyst. 2013, 138.17, 4982.

10. Buzzard, G. T.; Lucier, B. J. Computational Imaging. 2013, 865707.

11. Rehrauer, O. G.; Dinh, V. C.; Mankani, B. R.; Buzzard, G. T.; Lucier, B. J.; Ben-Amotz, D. Appl. Spectrosc. 2017. 0003702817732324.

12. Rehrauer, O.G.; Mankani, B.R.; Buzzard, G.T.; Lucier, B.J.; Ben-Amotz, D. 2015, Opt. Express, 23(18), pp.23935-23951.

13. Haka, A .S.; Shafer-Peltier, K.E; Fitzmaurice, M; Crowe, J.; Dasari, R.R; Feld, M.S. Cancer. Res. 2002, 62.18, 53755380.

14. Morgan, M.P.; Cooke, M.M.; McCarthy, G.M. Journal of mammary gland biology and neoplasia. 2005, 10.2, 181-187.

15. Breast Calcification: a Diagnostic Manual, A. Evans, Cambridge University Press, 2002

16. Kerssens, M. M.; Matousek, P.; Rogers, K.; Stone, N. Analyst, 2010, 135.12, 3156.

17. Baker, R.; Rogers, K. D.; Shepherd, N.; Stone, N. Br. J. Cancer, 2010, 103.7, 1034-1039.

18. Réfrégier, P.; Scotté, C.; De Aguiar, H. B; Rigneault, H.; Galland, F. J. Opt. Soc. Am. A. 2018, 35, 125-134

19. Berto, P.; Scotté, C.; Galland, F.; Rigneault, H.; De Aguiar, H. B. Opt. Lett. 2017, 42.9, 1696-1699.

20. Robbins, M. S.; Member, S.; Hadwen, B. J. IEEE Trans. on Electron Devices. 2003, 50.5, 1227-1232.

21. Hirsch, M.; Wareham, R. J.; Martin-Fernandez, M. L.; Hobson, M. P.; Rolfe, D. J. PLoS ONE. 2013, 8.1.

22. Harpsøe, K. B. W.; Andersen, M. I.; Kjægaard, P. Astron. Astrophys. 2012, 537, 1-12. 